\title{
Production de viande
}

de boucherie à partir

du cheptel laitier

Ath Agriculture

Canada

Publication 1456/F

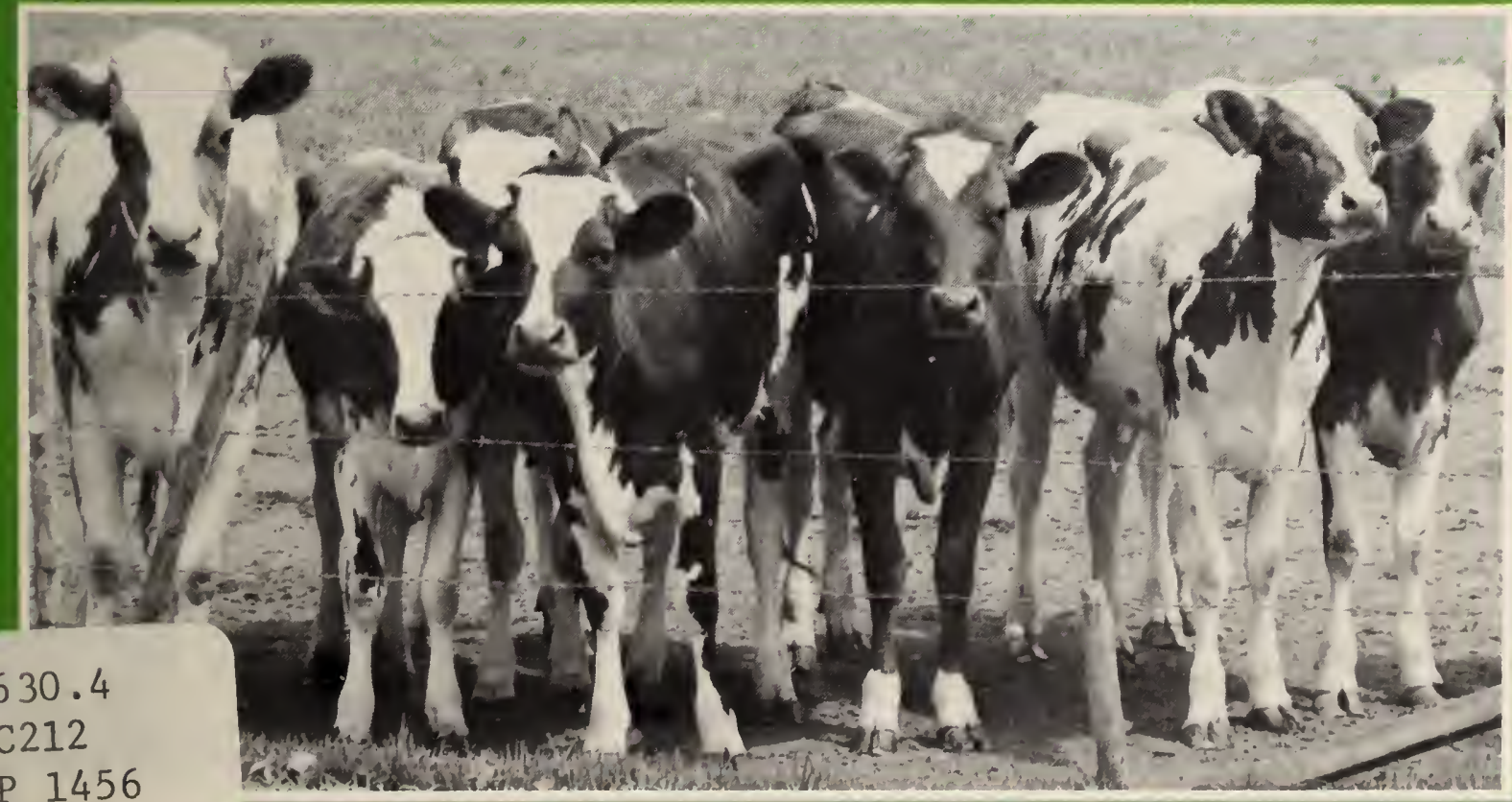

1986

fr.

$20 \mathrm{Ag}$

c. 3

Canadä 
Digitized by the Internet Archive in 2012 with funding from

Agriculture and Agri-Food Canada - Agriculture et Agroalimentaire Canada 


\title{
Production de viande de boucherie à partir du cheptel laitier
}

\author{
R.J. Forrest ${ }^{1}$
}

Station de recherches, Agassiz (C.-B.)

et

G.L. Roy

Station de recherches, Lennoxville (Qc) 
PUBLICATION 1456/F On peut en obtenir des exemplaires à la Direction générale des communications, Agriculture Canada, Ottawa K1A 0 C7.

${ }^{\circ}$ Ministre des Approvisionnements et Services Canada 1986

$\mathrm{N}^{\circ}$ de cat. A53-1456/1986F ISBN: 0-662-93599-3

Impression 1971 Révision $1986 \quad 2 \mathrm{M}-8: 86$

Also available in English under the title

Beef production from the dairy herd. 


\section{TABLE DES MATIÈRES}

Historique de l'utilisation de bovins laitiers comme source de viande de boucherie/ 6

Veaux/8

Comment se procurer des veaux?/8

Élevage par le producteur laitier/8

Élevage sous contrat par le producteur laitier/8

Élevage par un spécialiste/8

Achat de veaux à l'encan $/ 8$

Élevage des veaux/9

Santé du veau nouveau-né/9

Précautions et recommandations concernant l'élevage des veaux/9

Alimentation des jeunes veaux $/ 10$

Bouvillons/ 12

Alimentation des bouvillons/ 12

Infections et maladies fonctionnelles des bovins élevés dans les parcs

d'engraissement/12

Variations de l'efficacité alimentaire durant la croissance/13

Modifications de la carcasse durant la croissance/15

Effet des implants d'hormones/18

Alimentation composée entièrement de concentrés/20

Taureaux de race Holstein utilisés comme producteurs de viande de boeuf/22

Comparaison entre des bouvillons de race Holstein-Frisonne et des bouvillons de race Hereford/24

Conclusions/27

Bibliographie/27

Annexe Régime d'alimentation pour les bouvillons laitiers/28 


\section{HISTORIQUE DE L'UTILISATION DE BOVINS LAITIERS COMME SOURCE DE VIANDE DE BOUCHERIE}

Jusqu'à la fin du XIX $\mathrm{X}^{\mathrm{e}}$ siècle et au début du XX $\mathrm{XX}^{\mathrm{e}}$ siècle, la viande bovine au Canada était produite principalement par trois races britanniques de bovins, soit les Hereford, les Angus et les Shorthorn. L'élevage et la finition des animaux étaient fondés en grande partie sur des régimes de graminées et de fourrages conservés, et l'abattage avait lieu lorsque les animaux pesaient entre $600 \mathrm{~kg}$ et $900 \mathrm{~kg}$, à l'âge de 4-5 ans. Avec les années, cette pratique a changé progressivement, si bien que, à l'heure actuelle, les bovins de boucherie reçoivent des graminées jusqu'à ce qu'ils atteignent un poids variant entre $270 \mathrm{~kg}$ et $400 \mathrm{~kg}$, puis ils sont soumis à des régimes de finition dans des parcs d'engraissement jusqu'à ce qu'ils atteignent entre $445 \mathrm{~kg}$ et $550 \mathrm{~kg}$. Les régimes de finition sont composés principalement d'aliments concentrés.

Actuellement, l'industrie étudie encore une fois la possibilité de finir les bovins avec des rations contenant des proportions plus élevées de fourrages grossiers. Comme d'habitude, ce sont les conditions économiques qui gouvernent le genre exact des rations de finition utilisées par les éleveurs. Lorsque le prix du bœuf est bas et que celui des céréales est élevé par rapport à celui des fourrages, les éleveurs cherchent davantage à finir leurs bovins avec des fourrages. Il est probable que, à l'avenir, les éleveurs chercheront également à faire une utilisation plus intense d'aliments d'appoint tels que les déchets forestiers, le fumier de volaille, les déchets de conserveries et les rebuts horticoles pour finir les bovins de boucherie. Pour obtenir plus d'information sur les aliments de rebut, le lecteur pourra consulter la publication intitulée «Rebuts de pommes de terre pour l'alimentation des bovins», de Nicholson (1974).

En Amérique du Nord, la production de bovins de boucherie est liée en grande partie à l'utilisation de grands pâturages ne convenant pas à la culture. Par ailleurs, les bovins laitiers sont soumis à des conditions d'élevage et d'alimentation beaucoup plus intensives tout au long de l'année. Par conséquent, les deux industries, bovins de boucherie et bovins laitiers, ont connu un développement distinct en Amérique du Nord. Pour les éleveurs de bovins de boucherie, le phénotype idéal du bœuf de boucherie est un animal large et trapu, aux membres courts et à maturité précoce, tandis que les éleveurs de bovins laitiers préferrent un animal de grande taille, au corps anguleux, aux membres longs et à maturité plus tardive, ayant tout particulièrement de bonnes aptitudes à la production laitière. L'industrie du bœuf a interprété ces différences entre les deux types de bovins comme étant significatives de la qualité inférieure du bovin laitier en tant que producteur de viande. Des recherches ont montré que, bien qu'il existe des caractéristiques distinctives comme des différences de poids au même âge et une quantité plus élevée de gras interne inutile chez les bovins laitiers, celles-ci n'ont aucune influence sur la qualité ou le rendement de la carcasse. En fait, pendant des années, les mâles excédentaires provenant des troupeaux laitiers, soit environ $25 \%$ des veaux nés au Canada, ont servi à la production de viande de veau d'excellente qualité. De plus, toutes les vaches laitières trouvent le chemin de l'abattoir, où leur rendement en viande, principalement sous forme de bœuf haché ou de transformation, représente entre $30 \%$ et $40 \%$ de la production totale de bœuf. 
De nombreux rapports sur la possibilité d'utiliser les bovins laitiers pour produire des morceaux de bœuf de qualité (steaks et rôtis) ont été publiés depuis un certain nombre d'années. La première tentative importante visant à démontrer cette possibilité a été faite au Royaume-Uni dans les années 1950. Cela n’est pas étonnant, car le Royaume-Uni ne possède pas de grands pâturages pour la production du bœuf et est beaucoup plus dépendant de ses importations de bœuf que le Canada. Par conséquent, les chercheurs de ce pays ont décidé de tenter de remplacer une partie du bœuf importé par de la viande provenant des bovins mâles excédentaires de race Frisonne. Ils ont montré que l'on pouvait produire du bœuf d'excellente qualité en élevant des bouvillons de race Frisonne jusqu'à ce qu'ils atteignent environ $400 \mathrm{~kg}$, dans des conditions de claustration, de la naissance à l'abattage, et en leur donnant des rations composées à $85 \%$ d'orge et à $15 \%$ d'un complément fournissant un surplus de protéines, de minéraux et de vitamines. Depuis cette première expérience, de nombreux rapports de recherches sur la production de viande de bœuf avec des bovins laitiers mâles ont été publiés au Canada et à l'étranger.

Deux changements très importants se sont produits ces dernières années dans l'industrie canadienne du bœuf. Tout d'abord, il y a eu, depuis 1967, l'importation de races exotiques, la plupart originaires de l'Europe de l'Ouest, qui a permis l'implantation de 60 à 70 nouvelles races de bovins de boucherie au Canada. Ensuite, en 1972, le Canada a modifié ses normes de classement du bœuf. Les deux modifications les plus importantes étaient les suivantes : il devenait obligatoire de pratiquer une incision transversale entre les $11^{\mathrm{e}}$ et $12^{\mathrm{e}}$ côtes afin de mesurer l'épaisseur du grasं; de plus, les carcasses considérées d'excellente qualité devaient peser $272 \mathrm{~kg}$ (plus ou moins $45 \mathrm{~kg}$ ) et avoir entre $0,5 \mathrm{~cm}$ et $1,4 \mathrm{~cm}$ de gras subcutané sur la noix de côte. Ces normes ont été modifiées encore une fois en septembre 1984. Depuis ce temps, on pratique une incision transversale entre les $12^{\mathrm{e}}$ et $13^{\mathrm{e}}$ côtes, comme cela se fait aux États-Unis.

La principale différence qui existe entre un grand nombre des nouvelles races de bovins de boucherie et les races canadiennes implantées réside dans le fait que les premières ont un poids plus élevé, à la maturité, que les secondes. Par conséquent, les nouvelles races sont physiologiquement plus jeunes et ont moins de gras, au même poids d'abattage, que les races établies au pays. À cet égard, les races exotiques ressemblent à la race Holstein-Frisonne du Canada. La modification des normes de classement a été bénéfique pour les éleveurs de HolsteinFrisonne en ce sens qu'un nombre beaucoup plus élevé de carcasses de ces bovins se classent maintenant dans les catégories supérieures, Al et A2. La sélection pratiquée au sein des races établies afin qu'elles présentent les caractéristiques visées par les nouvelles catégories de classement a été également bénéfique car elle a permis de réduire la quantité de gras de rebut présente sur les carcasses de bovins finis.

La présente publication, fondée sur les travaux menés au Canada entre 1960 et 1985, renseigne sur les façons de produire de la viande de boucherie à partir du cheptel laitier. 


\section{VEAUX}

\section{Comment se procurer des veaux?}

\section{Élevage par le producteur laitier}

Le producteur laitier peut garder ses veaux mâles et les élever jusqu'à ce qu'ils aient atteint le poids du marché. Du point de vue de la santé des animaux, cette pratique est éminemment souhaitable. Le veau reçoit ainsi les meilleurs soins postnatals, comme le fait d'être nourri au colostrum. De plus, le taux de mortalité des veaux est généralement moins élevé lorsqu'ils demeurent sur la ferme où ils sont nés. Cependant, de nombreux producteurs laitiers préfèrent accroître leur capacité de production laitière plutôt que de se lancer dans l'élevage et la finition de bovins laitiers destinés à la boucherie. Ces producteurs sont davantage enclins à se départir de leurs veaux mâles rapidement et avec profit en les vendant à des particuliers ou à l'encan.

\section{Élevage sous contrat par le producteur laitier}

Un engraisseur-finisseur peut faire élever des veaux pour son compte dans une ferme laitière jusqu'à ce que les animaux aient atteint un âge (de préférence, après le sevrage) ou un poids précis. La valeur actuelle des veaux nouveau-nés et le coût de la main-d'œuvre et des aliments devraient permettre à l'engraisseur et à l'éleveur de vaches laitières de s'entendre sur un prix satisfaisant. Cette pratique d'élevage de bovins laitiers pour la boucherie a l'avantage de soumettre les animaux à une seule situation de stress, soit le transport.

\section{Élevage par un spécialiste}

Un spécialiste de l'élevage des veaux peut se procurer de jeunes veaux directement chez les éleveurs de troupeaux laitiers (ou dans les ventes à l'encan). Il les élève dans des conditions de contrôle rigoureuses et, dès qu'ils sont parvenus à un certain poids, il les revend à l'abattoir pour la production de viande de veau ou à un engraisseur-finisseur. Lorsque les veaux sont sevrés et qu'ils se nourrissent d'aliments secs, ils sont moins susceptibles de contracter des maladies. Cependant, même après le sevrage, les risques de tétanie de voyage et de pneumonie ne sont pas pour autant entièrement écartés. Il arrive souvent que le spécialiste de l'élevage des veaux continue d'engraisser ses veaux jusqu'à ce qu'ils aient atteint un poids marchand.

\section{Achat de veaux à l'encan}

De nombreux producteurs laitiers vendent à l'encan leurs veaux mâles excédentaires lorsque ceux-ci n'ont que quelques jours. Ainsi, chaque semaine, de grandes quantités de très jeunes veaux sont offerts sur le marché, bien qu'il y ait 
certaines périodes de l'année où les excédents sont moins importants. Toutefois, cette façon de procéder expose les veaux à contracter des maladies à un moment où justement ils sont le plus vulnérables. Le stress du déplacement, les refroidissements dus au froid et aux courants d'air, l'humidité et le manque temporaire de nourriture sont des facteurs qui contribuent à rendre les jeunes veaux davantage sujets à de graves problèmes de santé, ce qui peut entraîner des frais accrus de médicaments, une croissance retardée ou même la mort de l'animal. Lorsque l'on se procure des veaux de cette façon, il est conseillé de les tenir isolés pendant un certain temps avant de les intégrer au troupeau principal, afin de s'assurer qu'ils sont en bonne santé et qu'ils n'ont plus besoin de médicaments.

\section{Élevage des veaux}

\section{Santé du veau nouveau-né}

La santé des jeunes veaux est la principale préoccupation de l'éleveur de bovins laitiers de boucherie. La seule immunité dont ils jouissent jusqu'à l'âge de 3 ou 4 semaines leur vient du colostrum de leur mère. Les recherches ont établi que, pour acquérir une immunité passive contre les maladies, le veau doit recevoir du colostrum dans les douze heures qui suivent sa naissance. Malheureusement, exception faite d'une légère immunité générale qui lui est conférée par les protéines de son propre sang, le veau acquiert une immunité spécifique contre les seules maladies que la mère a contractées elle-même. Par conséquent, il s'ensuit qu'un veau retiré de son milieu habituel et exposé à d'autres sources de maladie a très peu de protection. En outre, d'autres facteurs combinés, comme le stress du déplacement et l'exposition au froid, aux courants d'air et à l'humidité, contribuent à rendre difficile l'élevage des veaux achetés dans les ventes publiques. Il faut s'attendre, dans ces cas, à des mortalités. Même avec les meilleurs soins, ces pertes sont généralement de l'ordre de 4 à $5 \%$, et occasionnellement de $15 \%$. La pneumonie ainsi que la diarrhée et les empoisonnements du sang sont des causes courantes de mortalités.

\section{Précautions et recommandations concernant l'élevage des veaux}

- Choisissez seulement des veaux robustes et sains. Les animaux pesant moins de $40 \mathrm{~kg}$ ont généralement plus de problèmes de santé. Assurez-vous que le nombril n'est pas enflé. N'achetez pas de veaux souffrant de diarrhée, qui ont les pattes raides ou des mucosités dans les narines ou qui respirent à un rythme rapide.

- Gardez les veaux dans un endroit chaud et sec. Si vous ne pouvez pas conserver une température de 15 à $21^{\circ} \mathrm{C}$ dans l'étable, installez une lampe chauffante pour chaque animal. Si les veaux sont élevés sur de la litière, ne la laissez pas devenir húmide.

- Mettez les veaux dans des enclos individuels ou des stalles entravées pendant les 5 à 8 premières semaines, afin de réduire les risques de transmission de maladies. 
- Assurez la ventilation du local à l'aide d'un ventilateur à commande thermostatique, de manière à ce que chaque veau dispose d'environ $23 \mathrm{dm}^{3}$ d'air par seconde. Il est nécessaire de prévoir des prises d'air à rainures au plafond pour l'arrivée d'air frais, afin d'éviter les courants d'air et de permettre à l'air frais de se réchauffer avant d'atteindre l'animal.

- Si les veaux souffrent de diarrhée, réduisez de moitié leur ration de lait ou d'aliment d'allaitement, mais ajoutez-y une quantité égale d'eau. On peut également donner aux veaux des solutions d'électrolytes afin de les empêcher de trop se déshydrater.

- Servez-vous d'un thermomètre rectal pour prendre la température de chaque veau à son arrivée, tous les deux jours, la première semaine, et ensuite seulement si l'animal respire à un rythme rapide, a des mucosités dans les narines ou souffre de diarrhée grave. Si la température du veau dépasse $39,7{ }^{\circ} \mathrm{C}$, consultez votre vétérinaire.

- Ne laissez pas les jeunes veaux se déshydrater s'ils souffrent de diarrhée grave ou persistante, car cela les rend extrêmement susceptibles aux infections respiratoires. Il est important d'établir un diagnostic et de commencer le traitement aussitôt que possible; aussi, consultez votre vétérinaire avant que la maladie n'ait trop progressé.

- Procédez à l'écornage et à la castration lorsque le veau est âgé de 3 à 6 semaines, à la condition toutefois qu'il soit en bonne santé. Plus le veau est âgé, plus il aura de la difficulté à s'en remettre. De toutes les méthodes disponibles, l'écornage pratiqué à l'aide d'un décorneur électrique et la castration faite à l'aide d'anneaux de caoutchouc ou par la méthode chirurgicale semblent provoquer un minimum d'inconfort pour le veau ou retarder sa croissance le moins possible.

- L'élevage en cases est une autre façon d'élever les veaux. Un certain nombre de producteurs laitiers qui ont des veaux ayant des problèmes de santé ont utilisé cette méthode avec succès. L'animal est placé dans une case (d'une grandeur approximative de $122 \mathrm{~cm} \times 122 \mathrm{~cm} \times 122 \mathrm{~cm}$ ) pourvue d'une litière et d'une ouverture sur un côté donnant accès à un petit enclos grillagé où t'animal peut faire de l'exercice. Avec cette méthode de gestion, il semble que les veaux ont moins de problèmes de santé, même par des températures inférieures à zéro. Les cases doivent être amovibles afin que l'on puisse les déplacer périodiquement pour empêcher une accumulation de bactéries pathogènes.

\section{Alimentation des jeunes veaux}

Chaque fois que cela est possible, il est préférable de se procurer des veaux qui ont reçu du colostrum après leur naissance. Le lait entier est encore le meilleur aliment à donner au veau, mais on peut obtenir de bons résultats avec des aliments d'allaitement commerciaux. Lorsque l'éleveur choisit d'utiliser ces derniers, il doit suivre avec soin le mode d'emploi recommandé par le fabricant. Les aliments commerciaux qui ont une teneur plus élevée en matière grasse et contiennent moins de produits céréaliers donnent généralement de meilleurs résultats, mais ils sont 
plus chers. Les sous-produits d'origine animale sont plus facilement consommés par le jeune veau (non ruminant).

Dès la première semaine, on devrait donner au veau une ration de démarrage de bonne qualité. Celle qui est présentée au tableau 1 a donné de bons résultats pour des veaux Holstein. Généralement, les rations de démarrage commerciales pour les veaux sont aussi efficaces et plus économiques que les mélanges préparés à la ferme. Il n'est pas recommandé de faire les mélanges à la ferme à moins que le nombre de veaux ne justifie l'achat de cinq tonnes d'aliments ou plus à la fois.

Des expériences ont montré qu'un veau qui commence tôt à se nourrir de céréales et qui continue d'en accroître sa consommation pendant l'étape de l'alimentation liquide a une meilleure performance et affiche le retard de croissance le moins important, peu importe qu'il soit sevré brusquement ou progressivement. Comme le lait ou les aliments d'allaitement sont habituellement plus chers que les aliments secs, on devrait envisager de sevrer les veaux assez tôt (entre 3 à 6 semaines d'âge). Durant cette étape, on peut donner du foin au veau. Toutefois, si l'éleveur prévoit mettre ses veaux à l'herbe ou leur donner d'autres fourrages après le sevrage, il devrait commencer à leur donner du foin à volonté aussitôt que possible. Cette pratique stimule le développement du rumen, permettant ainsi une utilisation complète des fourrages.

Certains éleveurs de veaux ont recours à l'alimentation par groupes en se servant de nourrisseurs automatiques. Il en existe plusieurs sortes sur le marché. Ces appareils mélangent une quantité prédéterminée d'aliment d'allaitement avec de l'eau chaude à intervalles réguliers et font parvenir le mélange jusqu'à une ou plusieurs tétines à proximité du veau. Ce système permet une économie considérable de main-d'œuvre, mais il occasionne davantage de problèmes de santé que le système d'alimentation individuelle.

\section{Tableau 1 Rations pour les bovins de race Holstein.}

\begin{tabular}{|c|c|c|}
\hline $\begin{array}{l}\text { Ration de } \\
\text { démarrage }\end{array}$ & $\begin{array}{l}\text { Ration de } \\
\text { croissance et } \\
\text { de finition }\end{array}$ & Prémélange \\
\hline
\end{tabular}

\section{Orge}

Avoine

Son

Tourteau de soja

Luzerne déshydratée

Prémélange

Farine d'os

Sel

Vitamine A (10 $000 \mathrm{UI} / \mathrm{g})$

Levure irradiée (170 000 unités de vitamine D par gramme)

Total

$\begin{array}{rrr}400 & 500 & - \\ 230 & 250 & - \\ 100 & 100 & - \\ 100 & 90 & - \\ 100 & - & - \\ 40 & 30 & - \\ 20 & 20 & 1 \\ 10 & 10 & - \\ - & - & 100\end{array}$

Protéines brutes approximatives

(en \%)

15,8

14.1 
On peut obtenir de bons résultats également en donnant aux veaux laitiers d'autres aliments comme du colostrum acidifié, du lactosérum ou du lait écrémé. Le lecteur pourra se renseigner davantage sur l'alimentation des veaux laitiers et les soins à leur donner en consultant les ouvrages mentionnés dans la bibliographie.

\section{BOUVILLONS}

\section{Alimentation des bouvillons}

On peut adopter, pour l'élevage des bouvillons laitiers, une variété de régimes d'alimentation, allant d'un régime extensif (fourrages seulement) à un régime intensif (aliments concentrés seulement). Les chercheurs de la station de recherches de Lennoxville ont mis au point un régime intermédiaire où l'on donne à l'animal à la fois des fourrages et des aliments concentrés (voir l'annexe). À la station d'Agassiz, les chercheurs ont mené des expériences visant à examiner la croissance et les caractéristiques des carcasses de bouvillons abattus à des poids vifs variant entre 161 et $835 \mathrm{~kg}$, à qui l'on avait donné surtout des rations composées en grande partie ou uniquement d'aliments concentrés. Ils ont également évalué l'utilisation des hormones comme moyen d'améliorer le rythme de croissance et la qualité de la carcasse de taureaux et de bouvillons de race Holstein (voir le chapitre intitulé «Effet des implants d'hormones»).

\section{Infections et maladies fonctionnelles des bovins élevés dans les parcs d'engraissement}

La santé des animaux, bouvillons ou veaux, est d'une importance primordiale. Les bovins laitiers, principalement les bouvillons de race Holstein-Frisonne, donnent peu d'ennuis dès qu'ils sont mis au régime sec et qu'ils pèsent plus de $130 \mathrm{~kg}$, pourvu qu'ils ne soient pas soumis à des conditions défavorables dans leur milieu. Une mauvaise ventilation, une humidité élevée ou une litière mouillée peuvent augmenter la fréquence et l'intensité des flambées de maladies. Dès qu'un animal a un comportement anormal, on doit l'isoler et lui donner le traitement médical approprié. Il est essentiel d'observer régulièrement l'état de santé des bouvillons afin de réduire les pertes financières attribuables à la mort de l'animal ou à une longue maladie. Le rétablissement de l'animal sera d'autant plus assuré que l'on aura décelé et traité rapidement ses problèmes de santé.

Les cas de météorisme sont plus fréquents lorsque les animaux reçoivent des régimes composés uniquement d'aliments concentrés. On peut atténuer ce problème en ajoutant à la ration des fourrages grossiers comme du foin ou des ensilages. Les animaux peuvent également souffrir de ruminite s'ils consomment seulement des aliments concentrés pendant de longues périodes. Les bouvillons cherchent à remédier à l'inconfort qu'ils ressentent en consommant une partie de leur litière fraîche, même si elle n'est faite que de copeaux. Bien qu'il soit possible d'élever des bouvillons, du sevrage à l'abattage, avec des rations entièrement composées de concentrés, il n'est généralement pas recommandé de le faire à moins que l'écart de prix entre les céréales et les fourrages grossiers ne soit 
exceptionnellement faible. Habituellement, les taux de gain pondéral ne diminuent pas lorsque l'on donne aux animaux des rations comprenant jusqu'à $40 \%$ de fourrages grossiers d'excellente qualité.

Il arrive parfois qu'un animal boite par suite d'une fourbure attribuable à un malaise digestif provoqué par une trop grande consommation d'aliments concentrés. En général, cette affection n'est pas mortelle, mais elle entraîne une réduction de la quantité d'aliments ingérée et du taux de gain pondéral. $\mathrm{Si}$ l'affection persiste, il est recommandé d'éliminer les animaux gravement atteints.

L'incidence de foies trop gravement atteints attribuable à la présence d'abcès chez les bouvillons de race Holstein est généralement peu élevée, se situant à moins de $5 \%$ des bovins abattus. Cette condition peut être provoquée par un changement brusque dans le régime de l'animal. On a déjà vu, à l'abattage, jusqu'à $70 \%$ et même $80 \%$ des foies trop gravement atteints après que les animaux furent passés de très grands pâturages à des parcs d'engraissement pour la finition en claustration. D'autres facteurs de stress, comme le transport, une manipulation fréquente des animaux, des combats entre eux, la surpopulation ou un changement de milieu, peuvent augmenter la fréquence des abcès du foie chez les bovins élevés dans les parcs d'engraissement.

On doit accorder une attention particulière aux quantités de vitamine A et, aussi peut-être, de vitamine D lorsque l'on donne aux bouvillons des rations composées en grande partie ou uniquement d'aliments concentrés. Dans un essai d'alimentation, quelques bouvillons et taureaux Holstein recevant une ration entièrement concentrée ont souffert de graves convulsions périodiques et de cécité à divers degrés. D'autres affections normalement attribuables à une carence en vitamine A, comme un larmoiement excessif des yeux, la diarrhée, des écoulements nasaux et des maladies pulmonaires, ne se sont pas manifestées. Des injections de vitamine $\mathrm{A}$ ont enrayé la progression de la cécité et éliminé les convulsions. À aucun moment de l'essai, avant ou après le traitement, les chercheurs n'ont constaté une baisse de la consommation d'aliments ou du taux de gain pondéral.

\section{Variations de l'efficacité alimentaire durant la croissance}

Dans des conditions normales, l'efficacité alimentaire, définie comme étant la quantité d'unités de matières nutritives nécessaires à un animal pour réaliser un gain déterminé de poids vif, augmente de la naissance jusqu'à la maturité. Cette augmentation constante est attribuable à l'accroissement des besoins d'entretien de la masse corporelle et des dépôts de gras. De toute évidence, un bouvillon de $500 \mathrm{~kg}$ a une masse corporelle dix fois plus volumineuse à entretenir qu'un veau de $50 \mathrm{~kg}$, et, par conséquent, le coût par kg de gain de poids est considérablement plus élevé dans le cas du premier que du second. Ce rapport est illustré au tableau 2. Ainsi, un bouvillon de $260 \mathrm{~kg}$ a besoin d'un total de 4,56 kg d'unités nutritives totales (U.N.T.) pour réaliser un gain pondéral de $1 \mathrm{~kg}$, tandis qu'un bouvillon de $620 \mathrm{~kg}$ a besoin de 7,64 kg de U.N.T. Une partie de cet écart peut être attribuée au fait que le bouvillon plus lourd amasse davantage de gras sur sa carcasse que le bouvillon plus léger. Tous les bouvillons ayant participé à l'essai d'alimentation dont il est question dans le tableau 2 ont reçu une ration de croissance (tableau 1), 
ainsi que du foin et de la pulpe de betterave pour les $100 \mathrm{~kg}$ de gain de poids avant l'abattage.

On peut voir également au tableau 2 que, sur une longue période, le taux de croissance est sigmoïde, c'est-à-dire en forme de S. À un poids d'environ $100 \mathrm{~kg}$, le taux de croissance est de $0,72 \mathrm{~kg}$ par jour; à un poids variant entre $180 \mathrm{~kg}$ et $700 \mathrm{~kg}$, le taux de croissance passe de 1,23 à 1,27 à 1,12 kg par jour; enfin, à un poids d'environ $780 \mathrm{~kg}$, à mesure que le bouvillon approche de la maturité, le taux de gain pondéral par jour diminue pour atteindre $0,81 \mathrm{~kg}$.

Ces données sur le taux de croissance et l'efficacité alimentaire sont caractéristiques aux bouvillons de race Holstein à qui l'on a fourni une alimentation riche en énergie de la naissance à l'abattage. Dans le cas des bouvillons nourris exclusivement avec des fourrages (grands pâturages, pâturages ordinaires, foin ou ensilage), les gains de poids sont moins élevés et les quantités d'unités d'aliments nécessaires par unité de gain pondéral sont plus élevées. Lorsque l'on change l'alimentation des bouvillons, passant des grands pâturages ou des fourrages à des rations de céréales à valeur énergétique élevée, on peut s'attendre à des taux de croissance aussi élevés que 1,5 kg par jour et même davantage.

Comme il a été mentionné dans les pages précédentes, il y a diverses façons de se procurer et d'élever des veaux de race Holstein. Les veaux qui ne sont pas destinés à la production de viande de veau sont engraissés jusqu'à ce qu'ils atteignent des poids plus élevés, convenant à la production de viande de bœuf. Une fois que l'engraisseur-finisseur a pris la décision de finir ses bouvillons laitiers pour la production de viande de bœuf, il doit garder à l'esprit les exigences du marché ou des exploitants d'abattoir concernant les bouvillons finis. Pour être classé dans la catégorie A1 ou A2, un bouvillon doit produire une carcasse pesant entre $250 \mathrm{~kg}$ et $317 \mathrm{~kg}$ et présentant une couverture de gras suffisante (entre $4 \mathrm{~mm}$ et $14 \mathrm{~mm}$ ). Par conséquent, le poids vif de l'animal doit se situer entre 435 et $550 \mathrm{~kg}$. L'éleveur obtiendra un prix moins élevé pour son animal si la finition est trop peu avancée ou trop avancée, même si le poids du bouvillon se situe dans la fourchette de poids établie. De même, il obtiendra des prix moins élevés pour des carcasses pesant moins de $250 \mathrm{~kg}$ ou plus de $317 \mathrm{~kg}$, même si elles ont une couverture de gras suffisante pour mériter une catégorie A1 ou A2.

\section{Tableau 2 Croissance et efficacité alimentaire des bouvillons Holstein.}

\begin{tabular}{ccccc}
$\begin{array}{c}\text { Intervalle } \\
\text { de } \\
\text { croissance } \\
(\mathrm{kg})\end{array}$ & $\begin{array}{c}\text { Gain de } \\
\text { poids } \\
\text { par jour } \\
(\mathrm{kg})\end{array}$ & $\begin{array}{c}\text { Quantité } \\
\text { d'aliments } \\
\text { par jour } \\
(\mathrm{kg})\end{array}$ & $\begin{array}{c}\text { Aliments } \\
\text { concentrés } \\
(\%)\end{array}$ & $\begin{array}{c}\text { U.N.T. } \\
\text { par kg de gain } \\
\text { de poids* } \\
(\mathrm{kg})\end{array}$ \\
\hline $\begin{array}{c}\text { 45-160 } \\
180-340\end{array}$ & 0,72 & 2,84 & 53 & 2,75 \\
$360-520$ & 1,23 & 8,59 & 58 & 4,56 \\
$545-700$ & 1,27 & 11,64 & 57 & 6,33 \\
$725-840$ & 1,12 & 12,41 & 60 & 7,64 \\
\hline
\end{tabular}

$*$ U.N.T. (unités nutritives totales $)=(0,5 \times$ foin $)+(0,75 \times$ aliment concentré $)$. 


\section{Modifications de la carcasse durant la croissance}

À mesure que l'animal croît, la composition du gain de poids se modifie. Au début, il y a une croissance rapide des os. Lorsqu'il est un peu plus vieux, on assiste à la formation rapide de viande maigre et, enfin, vers la fin de la croissance, ce sont surtout des dépôts de gras qui s'ajoutent à la carcasse. Une fois que l'animal a absorbé suffisamment d'aliments pour entretenir sa masse corporelle, le reste des aliments qu'il consomme sert à la croissance des os et à la formation des muscles et du gras. Si l'animal reçoit des rations ayant une faible teneur énergétique, par exemple lorsqu'il est élevé sur de grands pâturages, il ne se produit aucun engraissement notable jusqu'à ce que l'animal approche de la maturité. Cependant, l'engraissement débute beaucoup plus tôt si l'animal consomme des quantités importantes d'aliments à haute valeur énergétique comme des céréales ou du maïs. Ce fait est évident dans les données du tableau 3, où les animaux ont reçu jusqu'à $64 \%$ de leurs rations sous forme de concentrés. Avec un tel régime d'alimentation, les bouvillons avaient une couverture de gras suffisante pour se classer dans la catégorie Al quand ils avaient à peine plus de 9 mois et pesaient $339 \mathrm{~kg}$. Toutefois, les exploitants d'abattoir auraient offert un prix inférieur pour ces bouvillons, car

Tableau 3 Caractéristiques des carcasses de bouvillons ayant entre 161 et $835 \mathrm{~kg}$ de poids vif, à l'abattage.

Tranches de poids à l'abattage

\begin{tabular}{|c|c|c|c|c|}
\hline 1 & 2 & 3 & 4 & 5 \\
\hline 154 & 285 & 447 & 611 & 835 \\
\hline 161 & 339 & 520 & 704 & 835 \\
\hline 149 & 311 & 485 & 668 & 802 \\
\hline 7.5 & 8,3 & 6.7 & 5.1 & 4,0 \\
\hline 83 & 173 & 277 & 392 & 486 \\
\hline 55,7 & 55,6 & 57,1 & 58.7 & 60,6 \\
\hline 80 & 171 & 274 & 389 & 482 \\
\hline 3,6 & 1,2 & 1,1 & 0.80 & 0.82 \\
\hline 50,3 & 49.9 & 49,0 & 47.2 & 45,7 \\
\hline 2,4 & 2,6 & 8,6 & 21.2 & 61,4 \\
\hline 24,5 & 19.5 & 15,4 & 14.0 & 12,4 \\
\hline 7,6 & 20.8 & 31.5 & 41.2 & 43,0 \\
\hline 67,9 & 59.7 & 53.1 & 44.8 & 44.6 \\
\hline 0.9 & 2,9 & 5.5 & 9.2 & 15.2 \\
\hline - & 7.0 & 13.9 & 23.6 & 39.1 \\
\hline - & Al & $\mathrm{A} 2$ & A3 & A4 \\
\hline
\end{tabular}

* Extrait à l'éther du muscle longissimus dorsi (noix de côte). 

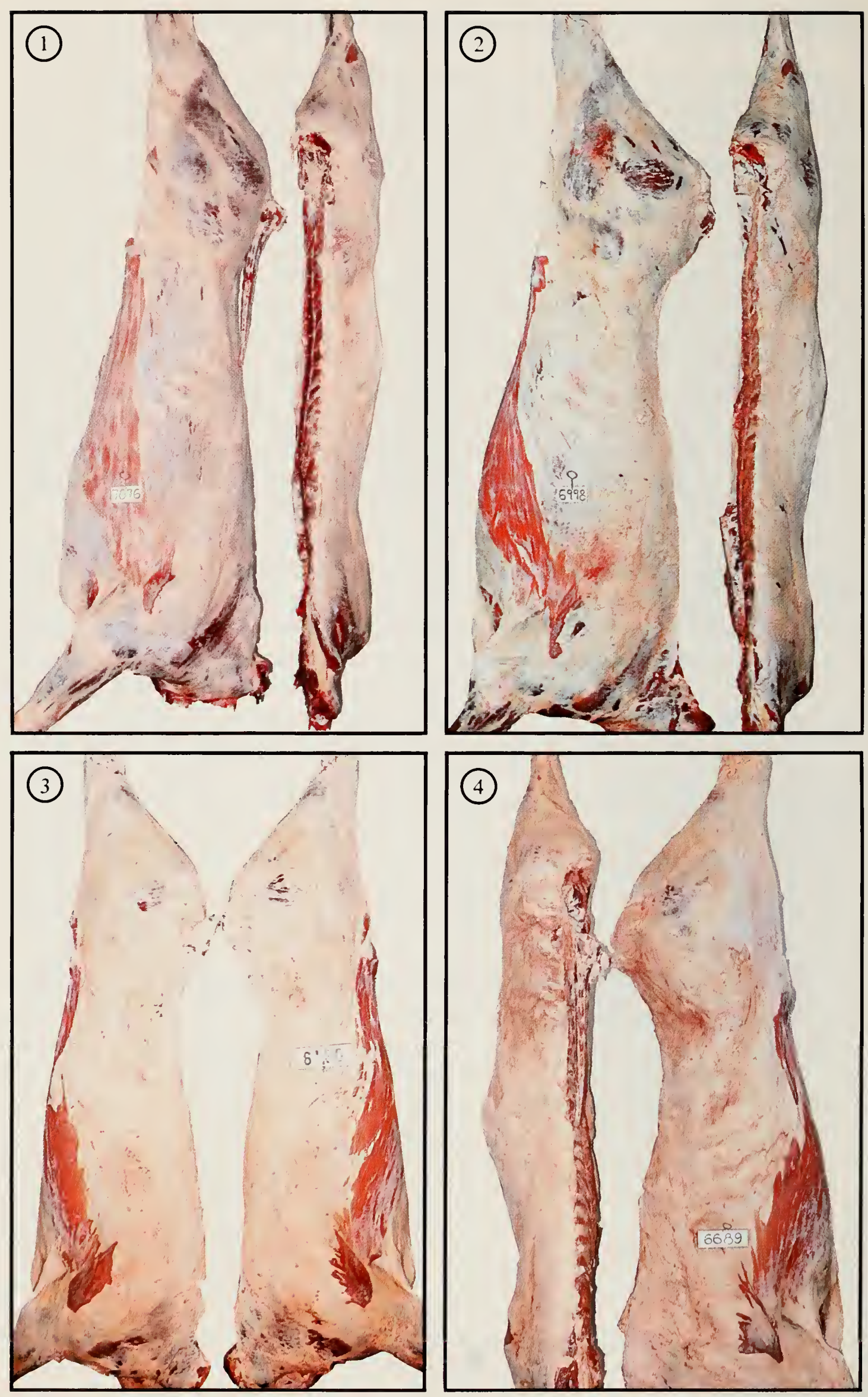
leurs carcasses ne pesaient que $173 \mathrm{~kg}$. À l'âge de 14,5 mois, au moment où leurs carcasses pesaient en moyenne $277 \mathrm{~kg}$, les bouvillons étaient entièrement acceptables et la plupart auraient été classés dans la catégorie A2. (Le prix payé par l'industrie est le même pour les carcasses de la catégorie A1 ou A2 dont le poids se situe dans la fourchette de poids jugée correcte.) À l'âge de 20 mois, les carcasses étaient trop lourdes et avaient une couverture de gras trop épaisse (catégorie A3). Enfin, au moment de l'abattage, à l'âge de 27,5 mois environ, les derniers bouvillons, pesant $802 \mathrm{~kg}$ vif, avaient une couverture de gras encore plus épaisse et ont été classés dans la catégorie A4.

D'un point de vue économique, il n'est pas pratique de nourrir des bouvillons Holstein avec des rations à valeur énergétique élevée jusqu'à ce que leur poids vif dépasse environ $600 \mathrm{~kg}$. Rendus à ce poids, les animaux ont une couverture de gras trop épaisse et leurs carcasses sont déclassées parce que pesant plus de $315 \mathrm{~kg}$. Cependant, les chercheurs ont mené cette expérience pour démontrer que l'on peut produire, avec des bovins de race Holstein, des carcasses excessivement grasses et de la viande fortement persillée (comme on peut le faire avec les bovins de boucherie); il faut noter toutefois que l'on doit élever les bovins laitiers jusqu'à ce qu'ils atteignent des poids à l'abattage supérieurs à ceux des bovins de boucherie, pour obtenir des carcasses comparables. Le persillage, ou pourcentage de gras (extrait à l'éther), de la noix de côte passait de 0,9 à 15,2\% pendant que le poids de l'animal passait de 165 à $802 \mathrm{~kg}$; le gras périrénal augmentait également pendant la même période, passant de 2,4 à 61,4 kg. De même, l'épaisseur du gras sur la côte augmentait de 7,6 à 43,0\% tandis que la proportion de viande maigre et celle d'os diminuaient, passant respectivement de 67,9 à $44,6 \%$ et de 24,5 à $12,4 \%$ (soit une diminution de $50 \%$ dans ce dernier cas). À mesure que les bouvillons Holstein engraissaient et parvenaient à maturité, les pourcentages de la perte de poids vif avant l'abattage et de la perte de poids de la carcasse ainsi que le pourcentage constitué par le quartier postérieur diminuaient de façon caractéristique tandis que le rendement à l'abattage augmentait. L'expérience a donc permis de conclure que, lorsque l'on finit des bouvillons Holstein avec des rations ayant une valeur énergétique relativement élevée, le poids idéal visé pour la finition est de $500 \mathrm{~kg}$. Les bouvillons qui reçoivent des rations ayant une valeur énergétique moins élevée doivent avoir un poids plus élevé à l'abattage et ont besoin d'une période de finition plus longue pour atteindre le même degré de finition.

\section{Effet des implants d'hormones}

À partir du milieu des années 1950 et pendant les années 1960 et 1970, le diéthylstilboestrol, hormone synthétique anabolisante, est devenu très prisé par les éleveurs pour sa capacité à stimuler le développement des animaux. On pouvait l'administrer oralement ou l'implanter sous la peau, dans l'oreille. Le traitement à cette hormone avait pour effet d'améliorer le taux du gain de poids et l'efficacité alimentaire, principalement chez les animaux élevés dans les parcs d'engraissement, et, souvent, d'augmenter la consommation quotidienne d'aliments. On ne connaît pas la façon dont le diéthylstilboestrol obtenait cette amélioration, mais, au bout du compte, il retardait le dépôt de gras et augmentait celui de protéines. Toutefois, il a été démontré que le diéthylstilboestrol avait des propriétés cancérigènes et son usage comme facteur de croissance a donc été interdit. À l'heure 
actuelle, il y a sur le marché d'autres composés anaboliques et antibiotiques qui ne sont pas cancérigènes et qui produisent les mêmes effets.

Deux hormones anaboliques, naturelles et femelles, et un produit chimique sont autorisés depuis un certain nombre d'années au Canada pour la croissance des bouvillons. Il s'agit du Synovex-S ${ }^{\circledR}$ ou du Steer-oid ${ }^{\circledR}$ (progestérone plus estradiol) et de Ralgro ${ }^{\circledR}$ (zéranol). Un troisième composé, le Compudose ${ }^{\circledR}$ (estradiol 17 beta), que l'on dit être un dérivé de l'estradiol à effets beaucoup plus prolongés que les autres implants, a été approuvé pour utilisation au Canada en 1984. Ajoutés à la ration, les ionophores Rumensin ${ }^{\circledR}$ (sodium de monensin) et Bovatec ${ }^{\circledR}$ (lasalocide) améliorent également le taux du gain pondéral ou l'efficacité alimentaire, ou les deux, présumément par un mécanisme différent, comme la modification des conditions du rumen. On fait aussi des essais (1985) sur trois autres ionophores, Avoparcin ${ }^{\circledR}, \operatorname{Narasin}{ }^{\circledR}$ et Salinomycin ${ }^{\circledR}$, afin de vérifier leur capacité à stimuler la croissance.

On recommande un autre implant combiné, Synovex-H ${ }^{\circledR}$ (estradiol et testostérone), pour améliorer le taux du gain pondéral et l'efficacité alimentaire chez les génisses. La testostérone est une hormone androgène et naturelle. Un autre composé, l'acétate de mélengestrol, améliore le rendement des génisses élevées dans les parcs d'engraissement en supprimant les périodes de rut.

Des expériences ont montré que l'implant d'hormones, Synovex-S ${ }^{\circledR}$, est efficace pour améliorer la croissance et l'efficacité alimentaire chez les bouvillons Holstein dont le poids vif se situe entre 181 et $726 \mathrm{~kg}$ (tableau 4). L'amélioration, en pourcentage, du gain pondéral a continué de s'accroître, passant de $13,8(181 \mathrm{~kg})$ à

Tableau 4 Effet, sur la croissance et l'efficacité alimentaire, des implants d'hormones administrés à des bouvillons Holstein de poids différents.

\begin{tabular}{|c|c|c|c|c|c|}
\hline $\begin{array}{l}\text { Poids au } \\
\text { moment de } \\
\text { l'implantation } \\
\quad(\mathrm{kg})\end{array}$ & Traitement & $\begin{array}{l}\text { Gain de } \\
\text { poids } \\
\text { par jour } \\
(\mathrm{kg})\end{array}$ & $\begin{array}{c}\text { Différence } \\
(\%)\end{array}$ & $\begin{array}{c}\text { Efficacité } \\
\text { alimentaire } \\
\text { (kg d'aliments } \\
\text { par kg de gain) }\end{array}$ & $\begin{array}{c}\text { Différence } \\
(\%)\end{array}$ \\
\hline \multirow{2}{*}{181} & Aucun & 1,15 & \multirow{2}{*}{13,8} & 4,76 & \multirow{2}{*}{9,4} \\
\hline & Implant & 1,31 & & 4,35 & \\
\hline \multirow{2}{*}{362} & Aucun & 1,08 & \multirow{2}{*}{31.5} & 7,01 & \multirow{2}{*}{24,1} \\
\hline & Implant & 1,42 & & 5,65 & \\
\hline \multirow{2}{*}{544} & Aucun & 0,96 & \multirow{2}{*}{33.1} & 8,35 & \multirow{2}{*}{17.1} \\
\hline & & & & & \\
\hline \multirow[b]{2}{*}{726} & Aucun & 0,81 & \multirow[b]{2}{*}{42,5} & 9.69 & \multirow[b]{2}{*}{18.2} \\
\hline & Implant & 1.15 & & 7.93 & \\
\hline
\end{tabular}


$42,5 \%$ ( $726 \mathrm{~kg})$. Cependant, la période la plus efficace pour obtenir une amélioration du gain total se situait lorsque l'animal pesait $362 \mathrm{~kg}$. En effet, à ce moment-là, le gain maximal passait de 1,08 à $1,42 \mathrm{~kg}$ par jour et l'amélioration de l'efficacité alimentaire était la plus élevée $(24,1 \%)$ chez les quatre groupes examinés. À des poids plus élevés ou moins élevés, l'amélioration de l'efficacité alimentaire était moins prononcée (de 9,4 à $18,2 \%$ ).

Au cours d'une autre expérience, on a montré les économies de concentrés qui ont été réalisées (remplacement des aliments concentrés par du foin) en faisant des implantations d'hormones sur des bouvillons Holstein (tableau 5). Durant une période de 167 jours, le groupe témoin nourri seulement avec des concentrés a consommé $1500 \mathrm{~kg}$ d'aliments concentrés et a réalisé un gain de poids de $197 \mathrm{~kg}$. Le groupe pourvu d'implants a consommé $1040 \mathrm{~kg}$ de foin et $749 \mathrm{~kg}$ de concentrés et a réalisé un gain de poids de $200 \mathrm{~kg}$. Le groupe témoin et le groupe pourvu d'implants ont produit des carcasses froides pesant respectivement 263 et $255 \mathrm{~kg}$. En supposant que le foin coûte $100 \$$ la tonne et les aliments concentrés coûtent $200 \$$ la tonne, la production de $1 \mathrm{~kg}$ de carcasse revient à $1,14 \$$ pour le groupe témoin et à $1,00 \$$ pour le groupe pourvu d'implants, ce qui représente une économie de 44,00 \$ par animal pour un coût approximatif de 3,00 \$ par implant.

\section{Tableau 5 Effet des implants d'hormones sur l'économie de la consommation d'aliments concentrés.}

$\begin{array}{cc}\text { Aucun } & \text { Implants } \\ \text { traitement } & \text { d'hormones }\end{array}$

Nombre de jours en parcs d'engraissement

\begin{tabular}{cc}
167 & 167 \\
1500 & 1040 \\
197 & 749 \\
& 200 \\
500 & 501 \\
263 & 255 \\
14,0 & 12,5 \\
$\mathrm{~A} 2$ & $\mathrm{~A} 2$ \\
$1,14 \$$ & $1,00 \$$ \\
\hline
\end{tabular}

Foin consommé (en kg)

Aliments concentrés consommés (en $\mathrm{kg}$ )

Variation dans le poids (en $\mathrm{kg}$ )

Poids vif à l'abattage, après perte de poids (en $\mathrm{kg}$ )

Poids de la carcasse froide (en $\mathrm{kg}$ )

Épaisseur du gras sur les côtes (en mm)

Catégorie de la carcasse

Coût des aliments par kg de carcasse*

* On suppose que le foin coûte $100 \$$ la tonne et les aliments concentrés, $200 \$$ la tonne.

\section{Alimentation composée entièrement de concentrés}

Avant les années 1950, on croyait qu'il fallait inclure, dans la ration des ruminants, des fourrages grossiers, comme du foin ou des graminées, en raison de leur teneur supposée en certains éléments nutritifs essentiels non connus. De nombreuses expériences menées depuis ces années-là sur l'alimentation entièrement concentrée ont montré que les fourrages grossiers exercent essentiellement une action physique plutôt que chimique. Une ration composée d'avoine aplatie et d'un supplément complet ou une ration mixte additionnée d'un supplément vitaminique (tableau 1) permet un taux de croissance élevé chez les bouvillons 
Holstein. Dans le cas d'une alimentation entièrement concentrée, il est important d'aplatir les céréales plutôt que de les broyer afin d'assurer le bon fonctionnement de l'estomac du bovin grâce à une quantité suffisante d'aliments grossiers. Si l'aliment concentré est composé uniquement de céréales broyées, il faut constituer une partie de la ration avec des fourrages grossiers afin d'assurer le bon fonctionnement du rumen. En outre, l'inclusion de fourrages grossiers dans la ration tend à réduire l'incidence de météorisme. Par conséquent, l'alimentation entièrement concentrée pour la production de viande à partir de bovins laitiers de boucherie est recommandée uniquement dans certaines circonstances spéciales, lorsque l'éleveur a tout le temps nécessaire pour observer les bouvillons et en prendre soin. Notons que l'on peut tout de même obtenir des gains maximaux lorsque la ration est composée à $20 \%$ de fourrages grossiers; en outre, ce genre de ration provoque moins de troubles digestifs que les rations entièrement concentrées. Les taux de gain pondéral sont réduits seulement lorsqu'on augmente la proportion de fourrages grossiers d'excellente qualité jusqu'au point où la capacité physique du rumen limite l'apport énergétique. Si l'on utilise des fourrages grossiers de moindre qualité, l'apport énergétique est limité plus rapidement étant donné que ce genre d'aliment nécessite une plus longue période de digestion dans le rumen.

On a mené également des expériences visant à comparer les effets des implants d'hormones chez des bouvillons nourris exclusivement de concentrés (tableau 6). On a donné aux bouvillons $160 \mathrm{~kg}$ de lait entier durant les huit premières semaines de leur vie, puis des aliments concentrés à volonté (tableau 1) jusqu'au moment de l'abattage, à un poids de $476 \mathrm{~kg}$. Les bouvillons pourvus d'implants ont atteint le poids prévu pour l'abattage 26 jours avant les sujets du groupe témoin. Une comparaison de toute la période de croissance, de la naissance à l'abattage, a également montré que le groupe pourvu d'implants avait engraissé plus vite $(6,8 \%)$, consommé moins d'aliments $(8,7 \%)$ et converti ses aliments plus efficacement $(9,3 \%)$ que le groupe témoin. Un examen de la seule période de traitement, soit celle où les animaux étaient pourvus d'implants et que leur poids passait de $340 \mathrm{~kg}$ à $476 \mathrm{~kg}$, a montré des résultats encore plus probants (de 18,7\% à $26,0 \%$ ). Les bouvillons du groupe témoin avaient $14,3 \%$ plus de gras, $4 \%$ moins d'os et $8,8 \%$ moins de viande maigre au niveau des côtes que ceux du groupe pourvu d'implants. Ces résultats illustrent l'effet principal des hormones qui est de diminuer ou de retarder le dépôt du gras et d'augmenter la proportion d'os et de viande maigre. Il n'est pas étonnant, par conséquent, que l'efficacité alimentaire soit améliorée, car $1 \mathrm{~kg}$ de viande maigre consiste en $25 \%$ de matières solides et $1 \mathrm{~kg}$ de gras consiste en $85 \%$ de matières solides environ, le reste étant constitué par de l'eau. De plus, le gras a une valeur énergétique qui est de 2,25 fois plus élevée que celle de la viande maigre. Par conséquent, $1 \mathrm{~kg}$ de gras contient environ 7,6 fois plus d'énergie que $1 \mathrm{~kg}$ de viande maigre.

Étant donné que le principal effet des implants d'hormones est de retarder le dépôt du gras, on peut s'attendre à ce que le moment le plus efficace pour implanter des hormones aux bovins Holstein soit lorsque ces derniers gagnent du poids rapidement, lorsqu'ils reçoivent uniquement des rations à haute valeur énergétique et qu'ils ont atteint le stade de l'engraissement ou de la finition. À tout autre stade de la croissance et pour n'importe quel autre régime d'alimentation, on peut s'attendre à ce que le traitement aux hormones soit moins efficace ou même ne produise aucun résultat. On peut également se demander si des implants d'hormones répétés 


\section{Tableau 6 Comparaison entre deux groupes de bouvillons nourris exclusivement d'aliments concentrés, dont un a reçu des implants d'hormones.}

$\begin{array}{lcc}\text { Groupe de } & \begin{array}{c}\text { Groupe de } \\ \text { bouvillons }\end{array} & \\ \text { bouvillons } & \text { traités aux } & \text { Différence } \\ \text { non traités } & \text { hormones } & (\%)\end{array}$

Poids à la naissance (en $\mathrm{kg}$ )

Poids vif à l'abattage, après perte de poids (en $\mathrm{kg}$ )

Gain de poids total (en $\mathrm{kg}$ )

Lait entier consommé (en $\mathrm{kg}$ )

Âge à l'abattage (en jours)

45

475

430

160

417

Taux de gain (en kg par jour)

Durant la période de traitement*

De la naissance à l'abattage

Aliments concentrés consommés (en $\mathrm{kg}$ )

Durant la période de traitement*

De la naissance à l'abattage

2481

5337
1,00

1,03

Efficacité alimentaire ( $\mathrm{kg}$ d'aliments $/ \mathrm{kg}$ de gain)

Durant la période de traitement*

De la naissance à l'abattage

Catégorie de la carcasse

5,62

A2

Composition des $9 \mathrm{e}, 10^{\mathrm{e}}$ et $11^{\mathrm{e}}$ côtes

(en \%)

Os

Gras

Viande maigre
17,8

33,6

48,6
477

430

160

391
47

\section{7}

. 列

.

1,26

1,10
26,0
6,8

2016

4872

18,7

8,7

(\%)

* Le traitement aux implants d'hormones a débuté lorsque les bouvillons ont atteint le poids de $340 \mathrm{~kg}$ et s'est poursuivi jusqu'à l'abattage, au moment où ils pesaient $476 \mathrm{~kg}$.

pendant l'étape de la croissance sont plus efficaces qu'un seul traitement appliqué au stade approprié de la croissance et de la maturité.

\section{Taureaux de race Holstein utilisés comme producteurs de viande de bouf}

Il est bien établi que les taureaux croissent plus rapidement que les bouvillons qui, à leur tour, se développent plus vite que les génisses. On sait également que les génisses atteignent leur maturité et engraissent plus précocement que les bouvillons qui, à leur tour, engraissent plus tôt que les taureaux. Il semblait donc logique d'examiner cette caractéristique, soit une croissance plus rapide, démontrée par les taureaux Holstein (mâles non castrés) afin d'établir si l'on pouvait en 
tirer profit pour produire de la viande de bœuf plus économique sans perte de qualité.

Des veaux mâles de race Holstein-Frisonne ont été mis sous observation dès leur naissance et ont reçu $150 \mathrm{~kg}$ de lait entier. La moitié d'entre eux ayant été castrés à l'âge de 4 semaines, l'expérience a donc porté sur 18 bouvillons et 18 taurillons (tableau 7). Dès la première semaine jusqu'à l'abattage, à un poids de $452 \mathrm{~kg}$, les animaux ont reçu uniquement des rations composées entièrement d'aliments concentrés (tableau 1). La ration de démarrage a été remplacée par une ration de croissance-finition lorsque les veaux ont atteint un poids de $135 \mathrm{~kg}$. Les taureaux ont atteint le poids fixé pour l'abattage 53 jours avant les bouvillons. Ils ont également gagné du poids plus rapidement $(15,5 \%)$, consommé moins de concentrés $(13,8 \%)$ et converti leurs aliments plus efficacement (14,2\%) que les bouvillons. Ces derniers avaient $32,7 \%$ plus de gras, 20,2\% moins de viande maigre et $6,3 \%$ moins d'os au niveau des côtes que les taureaux. Selon l'épaisseur du gras sur les côtes, les carcasses de bouvillons se seraient classées en moyenne dans la catégorie A2 et les carcasses de taureaux, dans la catégorie A1. L'expérience a donc permis de montrer que l'on pouvait produire de la viande de bœuf plus économique à partir de taureaux que de bouvillons. Étant donné que les taureaux étaient âgés de seulement 1 an au moment de l'abattage, ils n'étaient pas difficiles à conduire et ne présentaient pas un développement prononcé du cou comme les taureaux plus âgés. Aucune des carcasses ne présentait une viande à coupe sombre ou de couleur foncée au lieu d'une viande rouge vif. On a également constaté, au cours de cette expérience, que les taureaux vivant en groupe pendant un certain temps et ayant établi entre eux une hiérarchie sociale ne sont pas généralement difficiles à maîtriser et ne produisent pas une viande à coupe sombre

\section{Tableau 7 Comparaison entre des bouvillons et des taureaux de race Holstein, nourris exclusivement d'aliments concentrés.}

Bouvillons Taureaux Différence

$(\%)$

Nombre d'animaux

Poids à la naissance (en $\mathrm{kg}$ )

Poids vif à l'abattage, après perte de poids (en $\mathrm{kg}$ )

Âge à l'abattage (en jours)

Gain de poids par jour (en $\mathrm{kg}$ )

Aliments concentrés consommés (en $\mathrm{kg}$ )

$\mathrm{Kg}$ d'aliments concentrés par $\mathrm{kg}$ de gain

Poids de la carcasse chaude (en $\mathrm{kg}$ )

Rendement à l'abattage (en \%)

Épaisseur du gras sur les côtes (en mm)

Catégorie de la carcasse

Composition des $9^{e}, 10^{e}$ et $11^{e}$ côtes

(en \%)

Os

Gras

Viande maigre 
lorsqu'ils sont envoyés à l'abattage. Si des taureaux étrangers les uns aux autres sont regroupés peu de temps avant l'abattage ou au moment du transport vers l'abattoir, il peut se produire des combats entre ces animaux, et, souvent, un grand nombre d'entre eux produiront une viande de couleur foncée, empêchant les carcasses d'être classées dans les catégories supérieures.

Les rôtis provenant des taureaux et des bouvillons ayant participé à cette expérience ont été évalués par un groupe de dégustateurs experts. Les résultats obtenus ont montré que la viande provenant des deux groupes d'animaux était entièrement acceptable. Le consommateur ordinaire n'aurait pas pu faire la distinction entre la viande de taureau et la viande de bouvillon de cette expérience. La viande des 36 bovins ayant participé à l'expérience a été commercialisée localement, et aucune plainte n'a été reçue. De toute évidence, si des taureaux reçoivent une alimentation appropriée et sont abattus à une étape de la croissance pas trop avancée, ils peuvent produire à un coût moindre de la viande de bœuf d'une qualité similaire à celle produite par les bouvillons. En dépit de ces résultats, l'industrie bovine refuse encore, sans raison valable, de présenter de la viande de taureau au consommateur. On peut donc conclure qu'il faudra faire davantage d'efforts éducatifs en ce sens et davantage de travaux de recherches appliquées sur les divers aspects de la production de la viande de taureau avant que l'on puisse recommander cette pratique à tous les éleveurs.

Dans une autre expérience, on a également examiné les effets d'implants d'hormones sur des taureaux. Le traitement n'a produit qu'une très légère amélioration du taux de gain pondéral et de l'efficacité alimentaire. Par ailleurs, il a eu tendance à accroître le dépôt de gras plutôt qu'à le réduire, contrairement à ce que l'on obtient chez les bouvillons. Il faudra faire d'autres études scientifiques sur cet aspect de la production de viande de taureau. Il serait utile de trouver un traitement qui provoque le dépôt de gras, chez les taureaux, à un stade de la croissance plus précoce, sans pour autant diminuer le taux de gain ou l'efficacité alimentaire comme ce fut le cas dans cette expérience.

\section{Comparaison entre des bouvillons de race Holstein-Frisonne et des bouvillons de race Hereford}

Depuis toujours, l'industrie du bœuf soutient que la viande de bovins laitiers est de qualité inférieure à celle des races de boucherie. Elle prétend que le bovin laitier produit une viande moins bonne au goût et moins tendre et que sa carcasse a un rendement inférieur en viande vendable à cause de différences de conformation. Même aujourd'hui, les exploitants d'abattoir déclassent les carcasses de bovins laitiers et en offrent un prix inférieur. Ils classent la viande de bovins laitiers dans la catégorie A1X, bien que les inspecteurs d'Agriculture Canada la classent Al, catégorie la plus élevée du système canadien de classement du bœuf. Dans une large mesure, cependant, le prix plus bas reçu par les éleveurs de bovins laitiers de boucherie est compensé par le fait qu'ils peuvent se procurer des bovins laitiers d'engraissement à un prix moindre que les bovins de boucherie d'engraissement. La croissance des entreprises centralisées de dépeçage et de commercialisation du bœuf en carton devrait contribuer à éliminer la discrimination pratiquée par 
l'industrie entre les bovins à viande et les bovins laitiers de boucherie sur la base des différences de conformation. Du point de vue de la qualité au détail, la viande provenant de bovins laitiers est indifférenciable de la viande provenant de races établies de bovins de boucherie.

Les chercheurs des stations de recherches de Lennoxville et d'Agassiz ont comparé les caractéristiques des carcasses de bouvillons Holstein et Hereford. Dans l'expérience menée à Lennoxville, les bouvillons ont reçu une ration à valeur énergétique moyenne et ont été abattus lorsqu'ils ont atteint un certain poids, soit $543 \mathrm{~kg}$ dans le cas des Holstein et $443 \mathrm{~kg}$ dans le cas des Hereford (tableau 8). À ce stade, il y avait une différence de moins de $1 \%$ dans la teneur en gras au niveau de la $12^{\mathrm{e}}$ côte, entre les deux groupes. Toutefois, les bouvillons Holstein avaient près de trois fois plus de gras périrénal $(181,4 \%)$ et $3,7 \mathrm{~mm}$ de moins de gras $(29,1 \%)$ au niveau des côtes. On devrait abattre les bouvillons Holstein lorsque l'épaisseur du gras dorsal est minimale, afin que les bovins de cette race puissent faire concurrence aux races de boucherie comme les Hereford. L'abaissement des exigences du système canadien de classement des carcasses sur le plan de l'épaisseur du gras aurait des répercussions très favorables sur le classement des carcasses de bovins laitiers de boucherie.

À la station de recherches d'Agassiz, la comparaison entre les Holstein et les Hereford a porté sur des aspects différents. Il a déjà été démontré que les bovins de race Holstein-Frisonne produisent une quantité excessive de gras sous-cutané et de gras intramusculaire (persillage) (tableau 8), lorsqu'ils reçoivent une ration à haute valeur énergétique ou qu'ils atteignent un poids supérieur à $600 \mathrm{~kg}$. Par conséquent, les chercheurs ont décidé de comparer un certain nombre de caractéristiques des carcasses de Holstein ayant reçu des rations à haute valeur énergétique et des carcasses de Hereford ayant reçu des rations à valeur énergétique moyenne. Les deux groupes ont été abattus à des poids similaires, au moment où le pourcentage de gras au niveau des côtes était le même (tableau 9).

Tableau 8 Comparaison des caractéristiques de la carcasse entre des bouvillons Holstein et des bouvillons Hereford ayant reçu des rations à valeur énergétique moyenne.

Holstein Hereford Différence

$(\%)$

Poids vif final (en $\mathrm{kg}$ )

Poids de la carcasse chaude (en $\mathrm{kg}$ )

Rendement à l'abattage (en \%)

Gras périrénal (en $\mathrm{kg}$ )

Superficie de la noix de côte (en $\mathrm{mm}^{2}$ )

Épaisseur du gras sur les côtes (en mm)

Composition de la $12^{\mathrm{e}}$ côte (en \%)

Os

Gras

Viande maigre
543

292

54,4

16,6

64,2

8,4

17,4

26,0

55.9
443

235

54,5

5,9

62,0

12,1

15,9

26,2

57,1
22,6

24,3

0,2

181.4

3,5

29,1

9,4

0,8

2.1 
Lorsque les bouvillons Holstein et les bouvillons Hereford ont été privés de nourriture et d'eau pendant 24 heures, les premiers ont accusé une perte de poids plus élevée de 3,3\% et ont produit une carcasse ayant un rendement moins élevé de $2 \%$ et un quartier postérieur plus important de $0,5 \%$ que les seconds (tableau 9). Pour ce qui est du gras de rebut, les deux races ont accusé des différences substantielles, les Holstein ayant $54,2 \%$ plus de gras abdominal et $19,4 \%$ plus de gras périrénal. Cependant, elles ont montré une différence de moins de $0,2 \%$ pour ce qui est des proportions d'os, de gras et de maigre au niveau des côtes. Au total, les carcasses de Holstein avaient $1,3 \%$ plus d'os, $1,6 \%$ moins de gras et $0,3 \%$ plus de maigre que celles de Hereford. Les Hereford avaient plus de gras sous-cutané au niveau des côtes que les Holstein, mais la majorité des carcasses des deux groupes se sont classées dans la catégorie Al.

Les expériences qui ont été menées ont mis en lumière plusieurs différences et similitudes frappantes entre la race Holstein-Frisonne et la race Hereford. Il est évident que les Holstein accusent une perte de poids plus importante suivant une courte période de jeûne et qu'ils produisent une carcasse ayant davantage de gras de rebut et un rendement moins élevé que les Hereford. Par ailleurs, les carcasses des deux races ont essentiellement la même proportion de viande maigre et se classent dans les mêmes catégories. Par conséquent, il ne devrait pas y avoir de différence entre les deux races pour ce qui est des prix au crochet, mais les Holstein devraient justifier un prix inférieur s'ils sont vendus au poids vif, avant ou après la perte de poids. En supposant que le prix au crochet du bœuf est de 3,30 \$ le kilo (tableau 9), on peut déduire que les prix au poids vif avant perte de poids seront de 1,63 \$ et de

\section{Tableau 9 Comparaison des caractéristiques de la carcasse entre des bouvillons Holstein et des bouvillons Hereford.}

Holstein Hereford

Différence $(\%)$

Poids vif à l'abattage (en $\mathrm{kg}$ )

430

Poids vif à l'abattage, après perte de poids (en $\mathrm{kg}$ )

Perte de poids vif (en \%)

Poids de la carcasse chaude (en $\mathrm{kg}$ )

Rendement à l'abattage (en \%)

Quartier postérieur (en \%)

Gras abdominal (en $\mathrm{kg}$ )

Gras périrénal (en $\mathrm{kg}$ )

Composition des $9 \mathrm{e}, 10^{\mathrm{e}}$ et $11^{\mathrm{e}}$ côtes (en \%)

Os

Gras

Viande maigre

Composition totale de la carcasse (en \%)

Os

Gras

Viande maigre

Épaisseur du gras sur les côtes (en mm)

Catégorie de la carcasse
392

8,8
214

54,7

48,3

7,2

5,7

19,3

22,7

58,0

13,0

17.0

70,0

7,8

A I
1,6

5,3

3,3

8,9

2,0

0,5

54,2

$\begin{array}{ll}3,3 & 54,2 \\ 4,3 & 19,4\end{array}$

19,2

0,1

22,7

0.0

58,1

0,1

11,7

1,3

18,6

1,6

69.7

0,3

9,1

14.2

A1

- 
$1,75 \$$ le kilo et les prix au poids vif après perte de poids, de $1,78 \$$ et de $1,84 \$$ le kilo, pour les Holstein et les Hereford respectivement. Par conséquent, de justes écarts de prix entre les Hereford et les Holstein seraient de $0,0 \%$, de 3,3\% et de $6,9 \%$, selon que les prix sont fondés respectivement sur le poids au crochet, sur le poids vif après perte de poids et sur le poids vif avant perte de poids.

\section{CONCLUSIONS}

On peut produire de la viande de bœuf d'excellente qualité avec des bovins de race Holstein-Frisonne en utilisant les méthodes classiques d'élevage et de finition. Par ailleurs, le traitement aux implants d'hormones améliore le taux du gain pondéral et l'efficacité alimentaire en réduisant le dépôt de gras sans diminuer la qualité de la viande et permet au finisseur d'obtenir des profits additionnels. On peut également produire de la viande de bœuf plus économique avec des taureaux, mais cette viande est sujette à une certaine discrimination sur le marché. Les bouvillons de race Holstein accusent une perte de poids plus importante après un court jeûne et produisent des carcasses ayant davantage de dépôts de gras interne et un rendement moins élevé que les bovins Hereford, mais le prix des carcasses pour les deux races devrait être le même lorsque celles-ci sont classées, au crochet, dans les mêmes catégories.

\section{BIBLIOGRAPHIE}

Agriculture Canada, 1976, Système canadien de classement du bœuf, publication $\mathrm{n}^{\circ} 1579$ d'Agriculture Canada, 9 pages.

Hironaka, R. et Sonntag, B.H., 1982, Engraissement du bétail en parcs, publication no 1591 d'Agriculture Canada, 45 pages.

Ministère de l'Agriculture et de l'Alimentation de la Colombie-Britannique, 1981, Feeding and management guide for growing beef cattle, Ministère de l'Agriculture et de l'Alimentation de la Colombie-Britannique, Victoria (C.-B.).

Nicholson, J.W.G., 1975, Rebuts de pommes de terre pour l'alimentation des bovins, publication $\mathrm{n}^{\circ} 1527$ d'Agriculture Canada, 20 pages.

Winter, K.A., 1981, Sevrage à trois semaines et alimentation des veaux laitiers au colostrum fermenté, publication no 1721 d'Agriculture Canada, 9 pages.

Winter, K.A. et Lachance, B., 1983, Conduite et alimentation des jeunes bovins laitiers, publication no 1432 d'Agriculture Canada, 29 pages. 


\section{RÉGIME D'ALIMENTATION POUR LES BOUVILLONS LAITIERS}

On peut donner différents genres de rations aux bouvillons laitiers une fois qu'ils ont atteint un poids de $130 \mathrm{~kg}$. On doit viser un gain de poids de $1 \mathrm{~kg}$ par jour environ. On doit fournir un apport quotidien d'au moins $2 \mathrm{~kg}$ d'aliments concentrés afin que l'animal maintienne le taux de croissance désiré et atteigne le poids prévu pour l'abattage vers l'âge de 18 mois. Durant l'été, les bouvillons laitiers peuvent être mis à l'herbe. Des fourrages d'excellente qualité forment la base de rations économiques durant le stade de croissance. Un apport en céréales est nécessaire pour augmenter la valeur énergétique du régime et assurer ainsi une croissance continue.

On obtient des gains de poids d'environ $1 \mathrm{~kg}$ par jour lorsqu'on donne entre $2 \mathrm{~kg}$ et $3 \mathrm{~kg}$ de céréales par jour aux veaux du printemps mis dans de bons pâturages. Les recherches menées à Lennoxville ont montré que l'on obtenait la meilleure efficacité alimentaire, pour une ration composée de graminées et de tourteaux de céréales, lorsque l'on donnait à l'animal $2 \mathrm{~kg}$ de céréales par jour au lieu de $1 \mathrm{~kg}$ ou de $3 \mathrm{~kg}$. L'efficacité alimentaire accusait une amélioration de $25 \%$ ou plus dans le cas des animaux recevant $2 \mathrm{~kg}$ de céréales, par rapport à ceux qui en recevaient 1 ou $3 \mathrm{~kg}$. Par conséquent, il n'est pas recommandé de donner des céréales à volonté aux jeunes veaux en croissance mis au pâturage parce que le gain de poids supplémentaire n'est pas économique.

Au cours du premier hiver, on doit donner aux veaux tout le fourrage de bonne qualité qu'ils peuvent manger, plus 2,2 kg de céréales par jour. Habituellement, le bouvillon laitier ne tire profit que d'une seule saison de pâturage. Lorsque le bouvillon atteint le poids de $300 \mathrm{~kg}$ vif, on peut doubler sa ration quotidienne de concentrés afin d'augmenter les gains de poids quotidiens et de commencer le processus d'engraissement. À ce stade-ci, les rations devraient être constituées par des aliments à haute valeur énergétique tels que des ensilages de maïs, des ensilages de grains de céréales, du maïs d'une teneur élevée en humidité, du maïsgrain sec, de l'orge ou des pommes de terre de rebut. Enfin, durant le stade de l'engraissement (de $400 \mathrm{~kg}$ à $500 \mathrm{~kg}$ ), la ration peut être composée uniquement de grains. 




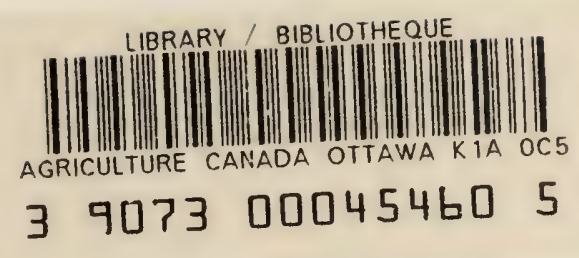


